\title{
干细胞的磁性标记研究进展
}

\author{
叶德文，王琪炜，张卫国，孙剑飞，顾宁* \\ 东南大学生物科学与医学工程学院, 南京 210096 \\ * 联系人, E-mail: guning@ @seu.edu.cn \\ 2017-04-28 收稿, 2017-05-27 修回, 2017-05-31 接受, 2017-06-22 网络版发表 \\ 国家自然科学基金(61420106012)资助
}

\begin{abstract}
摘要干细胞在组织修复与再生医学中具有广阔的前景, 但是千细胞体内移植后分布、活性、分化方向、作用 机制等认知的缺乏成为制约千细胞治疗发展的主要瓶颈，因此，千细胞体内示踪技术的发展对于解决上述问题将 起到至关重要的作用。目前利用磁性物质对干细胞进行标记后，结合磁共振成像技术(magnetic resonance imaging, MRI) 可以实现体外无创、安全、持续、动态的示踪观察，示踪的效果取决于细胞内所携带磁性物质的含量、不同 的磁性标记方式、细胞活性的维持. 本文将对千细胞磁性标记的不同方式、磁性物质的胞内代谢及对千细胞的影 响等研究进展进行系统综述，并结合现有的标记技术对如何提高干细胞磁性标记效率进行展望.
\end{abstract}

关键词干细胞示踪, 干细胞标记，磁共振成像，磁性氧化铁纳米颗粒，胞内铁代谢

干细胞是一类具有自我更新与分化功能的多能 细胞, 体内移植后可实现损伤组织或器官的再生 ${ }^{[1,2]}$. 已有研究报道, 干细胞可用于脊髓、大脑、肝脏等器 官损伤的临床治疗, 并且显示良好的治疗效果 ${ }^{[3 \sim 5]}$. 移 植干细胞在体内的定位、活性与分化等情况是对干细 胞治疗安全性与有效性的重要评估, 而归巢和迁移方 向的偏差被认为是干细胞治疗中存在的主要问题 ${ }^{[6,7]}$, 因此需要长期持续有效且无创的体外跟踪监测手段. 磁共振成像技术(magnetic resonance imaging, MRI)相 对于超声 (ultrasound, US)、电子计算机断层扫描 (computed tomography, CT)、正电子发射型计算机断层 显像(positron emission tomography, PET)等其他医学 影像方法具有无辐射、信号穿透衰减小、空间分辨率 高与组织对比度大等优点 ${ }^{[8]}$. 超顺磁氧化铁纳米粒子 (superparamagnetic iron oxide nanoparticles, SPION) 在 生物医学领域有着广泛应用, 包括药物递送 ${ }^{[9]}$ 、细胞 分选 ${ }^{[10]}$ 与肿瘤热疗 ${ }^{[11]}$ 等, 并且由于其具有生物相容性 好、对比度强等特点成为目前最常用于细胞标记的
MRI示踪剂 ${ }^{[12,13]}$. 尽管如此, SPION应用于干细胞示踪 的临床试验依然面临着许多亟待解决的问题, 例如如 何在不影响干细胞活性的同时提高SPION对干细胞的 标记效率, 如何缓解由于胞内降解与细胞分裂所引起 的SPION体内稀释等. 近年来的相关研究正在逐步破 解这些难题, 本文将重点针对干细胞的磁性标记方式 以及细胞与SPION的相互作用等方面展开综述.

\section{1 干细胞的磁性标记方式}

用于示踪的干细胞磁性标记方式主要分为两种: 一种是直接标记，通过细胞摄人SPION使其携带磁 性物质; 另一种标记方式为间接标记，通过基因转染 使细胞表达特定的蛋白质，增强弛豫率及组织对比 度, 提高 $\mathrm{T} 1$ 或 $\mathrm{T} 2$ 加权成像.

\subsection{SPION标记干细胞}

\subsection{1 细胞对SPION的摄入机制}

细胞对 SPION的摄人过程分为两个过程：一是 
细胞膜与颗粒的接触, 二是颗粒由细胞膜向胞内的 转运. 其中转运过程主要由网格蛋白和小窝蛋白介 导, 小窝蛋白尺寸为 60 80 nm, 不适于大尺寸颗粒 的转运, 相比之下网格蛋白介导的内吞方式更为广 泛, 除此之外还有细胞膜穿孔内吞、转运肽介导的直 接内化与巨胞饮作用等转运方式 ${ }^{[14,15]}$. 细胞的标记 效率不仅取决于细胞的性质 (膜的特性以及细胞大 小), 还受到颗粒性质(尺寸、形状、分散性、表面电 荷)的影响 ${ }^{[16]}$. 比如, 颗粒的大小很大程度上影响了 颗粒进入细胞的途径, 有研究表明 ${ }^{[17]}$, 直径大于 60 $\mathrm{nm}$ 的纳米颗粒需要通过与大量受体结合后才可以驱 动膜包裹的过程, 但同时会限制其他纳米颗粒与膜 的结合; 直径小于 $30 \mathrm{~nm}$ 的纳米颗粒只有与大量受体 紧密聚集才能驱动膜包裹过程; 而直径30 60 $\mathrm{nm}$ 的 纳米颗粒能够高效地驱动膜包裹过程. 此外, 颗粒的 形状对吞噬也有一定影响, Zhang等人 ${ }^{[18]}$ 研究表明细 胞对球形颗粒相比圆柱形颗粒有着更强的吞噬效果. 对于一些吞噬性的细胞, 如巨噬细胞、中性粒细胞等, 采用颗粒与细胞简单的共孵育就能够实现有效标 记 $^{[19 \sim 21]}$, 对于干细胞及其他内吞能力较弱的细胞, 采用颗粒表面化学修饰、物理场介导作用等方法可以 增强标记效率同时保持细胞原有活性.

\subsubsection{SPION的表面修饰提高标记效率}

SPION标记干细胞时材料本身往往存在两个方 面的问题: 一是SPION不稳定, 颗粒间容易形成聚 集; 二是SPION与细胞膜间的相互作用弱. 使用一些
具有生物相容性的材料对 SPION表面进行修饰能够 提高颗粒的稳定性、增强颗粒与细胞的接触. 目前广 泛使用聚合物 (聚乙二醇、多聚糖、聚乳酸)、抗原抗 体、细胞穿透肽、无机材料硅 ${ }^{[22,23]}$ 等对SPION进行修 饰结构, 作用方式如图1所示.

在SPION表面修饰抗原抗体是一种蛋白之间特 异性结合的标记方式 ${ }^{[24]}$, 磁性颗粒上携带的配体能 够靶向性地与细胞膜上的受体结合, 引起局部细胞 膜内陷形成小泡, 增加细胞对 SPION的摄人. RGD三 配体、叶酸、转铁蛋白、肝素 ${ }^{[25,26]}$ 等配体连接在磁性 颗粒表面可以靶向表达特定受体的细胞, 使得 SPION在细胞的含量提高3 4倍, 标记量很大程度上 取决于细胞表面受体的表达情况 ${ }^{[27]}$. SPION还能够与 一些蛋白质结合进而促进细胞吞噬, HIV-1肽蛋白是 一种能够自由通过细胞核核膜的物质, 可以将颗粒 直接转运到细胞内 ${ }^{[28,29]}$. 最早, Weissleder研究组 ${ }^{[30]}$ 合成了肽链来修饰葡聚糖包被的 SPION, 紧接着 Jackson等人 ${ }^{[31]}$ 将肽修饰的SPION标记间充质干细胞 (mesenchymal stem cells, MSC), 移植到大鼠纹状体 中实现了 MRI的长时程观察. 然而蛋白质的修饰可 能会引起体内的免疫反应, 针对这个问题, 使用从细 胞本身获取的ATP对颗粒进行修饰可以减弱免疫反 应带来的影响, 由于ATP的一个部分对细胞膜的配 体有很强的针定作用, 并且它还具有用于与生物活 性分子缀合的偶联部分, 在成像示踪上能够起到靶 向探针的作用 ${ }^{[32]}$.

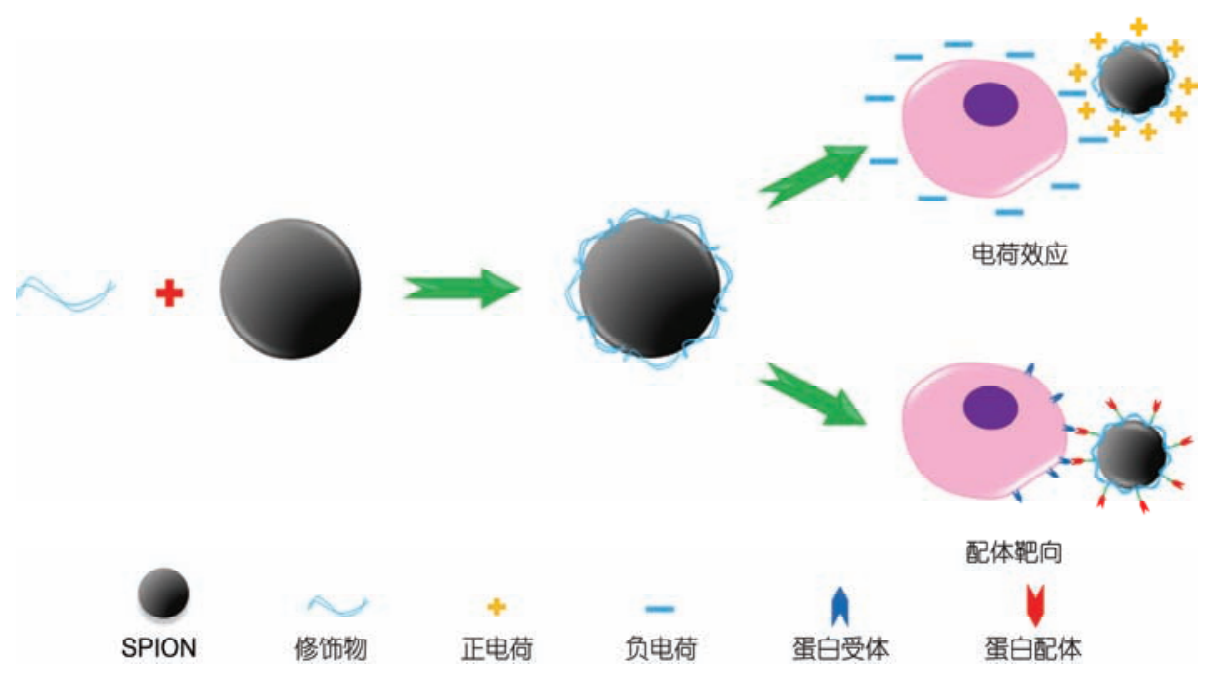

图 1 (网络版彩色)SPION表面修饰原理及作用方式

Figure 1 (Color online) Principle and modes of SPION surface modification 
颗粒与细胞表面的电荷作用也是影响细胞吞噬 颗粒的重要因素, 通过聚阳离子的转染试剂 (transfection agents, TA)涂覆表面能够使SPION表面携带 正电荷, 非特异性接触表面带有负电荷的细胞, 从而 通过电荷效应增加标记量 ${ }^{[33]}$. Bulte等人 ${ }^{[34]}$ 提出使用 树枝状聚合物修饰SPION能够增加干细胞标记量; 随后与Frank等人 ${ }^{[35]}$ 合作, 利用多聚赖氨酸( poly- $L-$ Lysine, PLL)、硫酸鱼精蛋白(protamine, PRO)等聚阳 离子试剂涂覆 SPION, 通过颗粒与细胞间静电吸附 作用实现了单个细胞中 $30.1 \mathrm{pg}$ 铁含量的标记量. 相 比使用单一材料涂覆 SPION, 两种材料的结合可以 起到相辅相成的效果, 肝素和鱼精蛋白可以通过静 电作用形成体内稳定的复合物 ${ }^{[36]}$, 同时证实肝素与 鱼精蛋白的不同比例混合修饰SPION对细胞的标记 效率具有显著影响 ${ }^{[37]}$. Kim等人 ${ }^{[38]}$ 在SPION表面修饰 了带有强正电荷的2-氨基乙基-三甲基铵(TMA), 对 人源间充质干细胞(human derived mesenchymal stem cells, hMSC)进行标记, 颗粒吞噬量大大提高. 不同 于阳离子转染剂, 最近有研究组制备了由柠檬酸盐 包被的SPION，柠檬酸盐包被的SPION显示带负电 荷, 可能是电荷形成的非特异性相互作用使细胞与 颗粒之间产生强亲和力, 对 $\mathrm{hMSC}$ 实现了 69.6 \pm 5.1 $\mathrm{pg} / \mathrm{cell}$ 的标记量 ${ }^{[39]}$. 上述的转染试剂能够有效地增 加标记浓度, 但是潜在的生物毒性不容忽视, 无机材 料如二氧化硅生物相容性好、更安全, 因此也常作为 涂覆材料 ${ }^{[40,41]}$.

然而基于膜表面受体的内化增强只适用于表达
特异性抗原/抗体的细胞, 并且存在体内免疫、趋向于 定位在细胞核等风险. 而有机聚合物对细胞具有潜 在毒性, 其毒性大小与使用的转染剂浓度成正比 ${ }^{[42]}$. 另外，共孵育的方式所需时间长，不适于临床应用. 考虑到上述问题，不使用转染剂的标记方式将更具 有临床应用价值，因此物理场介导的SPION摄人增 强机制以其安全、快速、可调的优势成为目前广受关 注的标记方法.

\subsection{3 物理场介导的颗粒摄入量增加}

物理场介导的颗粒摄人量增加是指在颗粒与细 胞共孵育时无需外加转染试剂等材料, 取而代之的 是对其施加电场、声场、磁场等物理场，可以在增强 标记量的同时缩短标记时间. 研究目前主要集中在3 种作用: 电脉冲穿孔 (magnetoelectroporation, MEP)、 超声脉冲穿孔 (magnetosonoporation, MSP)、磁场作 用，原理如图2所示:

$\mathrm{MEP}$ 是指在颗粒与干细胞共孵育时施加瞬时的 电脉冲波刺激干细胞, 使干细胞的膜通透性由于电一 机械作用发生瞬时改变，使细胞外大分子纳米颗粒 等物质内化. 早在 20 世纪 90 年代, 就出现了利用电脉 冲将DNA等大分子物质转运到细胞中用于基因转染. Bulte研究组 ${ }^{[43]}$ 利用电脉冲 $(75 \sim 400 \mathrm{~V}, 0.3 \sim 30 \mathrm{~ms})$ 对 鼠来源的神经干细胞(neural stem cell, NSC)进行标 记, 对细胞作用较低电压 $(40 \mathrm{mV})$ 、多个脉冲 $(n=10)$ 后发现标记量增大 2 倍. 此后, 他们对白细胞、淋巴细 胞进行了磁标记, 这两种细胞由于细胞质空间小难 以标记, 利用同样方式也观察到了吞噬量的增加, 进
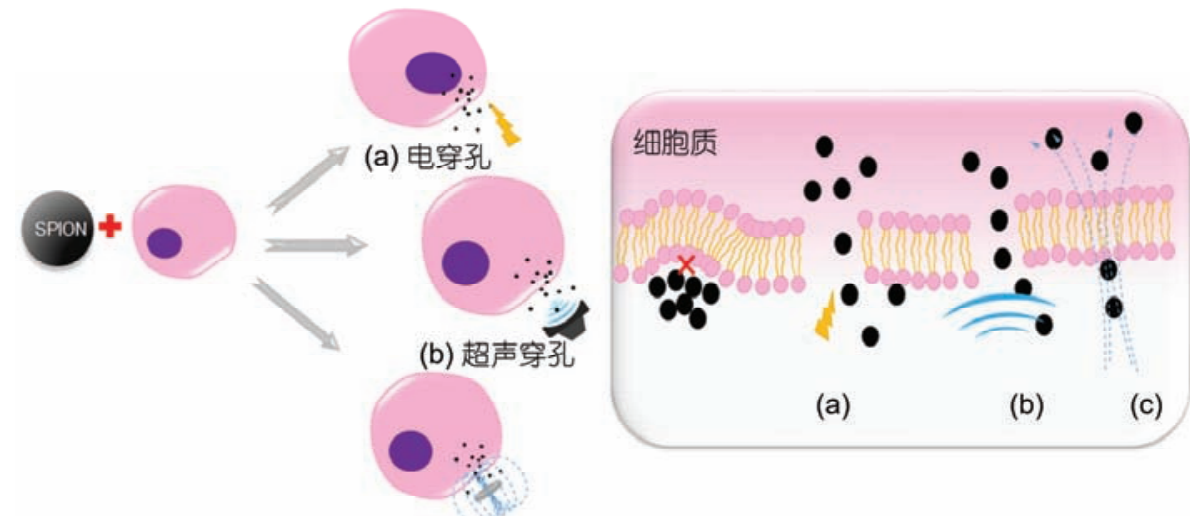

(c) 磁场作用

图 2 (网络版彩色)物理场介导的细胞标记. (a) 电穿孔促进细胞标记; (b) 声脉冲刺激促进的细胞标记; (c) 静、脉冲磁场作用的细胞标记 Figure 2 (Color online) Physical field mediated cell labeling. (a) MEP mediated cell labeling; (b) MSP mediated cell labeling; (c) static/pulsed magnetic field mediated cell labeling 
一步说明了MEP的有效性 ${ }^{[44]}$. 最近, Kim等人 ${ }^{[45]}$ 利用 电穿孔对脂肪间充质干细胞 (adipose derived mesenchymal stem cells, ADMSC)进行标记, 合成了一种氧 化硅涂覆的中空氧化锰纳米颗粒, 颗粒与干细胞共 孵育时对其施加脉冲电场作用(电压 $100 \mathrm{~V}$, 脉冲波 个数 $n=5$, 占空比 $95 \%$ ), MRI结果显示相对于普通孵 育, 电脉冲穿孔具有更短的 $\mathrm{T} 1$ 时间. 研究表明施加 的电刺激根据细胞、颗粒的特性决定, 电刺激过大会 导致细胞的死亡.

MSP是通过超声波作用, 瞬时可逆地改变细胞 膜的通透性. 对于声穿孔的机制研究, 普遍认为细胞 所处的培养环境(血液、培养液)中都含有微小气泡, 在超声作用时气泡的收缩振荡使细胞膜通透性增大. 最早, Bao等人 ${ }^{[46]}$ 观察到卵巢细胞在低声作用压下细 胞膜对大分子暂时打开, 而后一段时间内自动闭合. 此后, 利用超声空化进行细胞给药的治疗研究广泛 开展, $\mathrm{Qiu}$ 等人 ${ }^{[47]}$ 首次利用超声对神经干细胞 C17.2进 行了磁标记, 对细胞作用 $5 \mathrm{~min}$ 后标记量达到 5.22 pg/cell, MRI成像观察到小鼠体内明显的信号增强. 该研究组 ${ }^{[48]}$ 之后对超声参数进行了优化, 通过对比 不同强度、占空比的超声得到最适参数, 标记量达到 8 pg/cell. Lei等人 ${ }^{[49]}$ 利用“封闭”的超声发射装置对神 经干细胞C17.2进行了磁标记, 相比开放的装置, 该 设计能够减少细胞污染的损失, 细胞标记量可以达 到 $26.8 \mathrm{pg} / \mathrm{cell}$, 并且较低的占空比更适用于细胞标 记, 高的占空比会导致作用间隙过长的 SPION从细 胞中释放. 气泡作为一种介质是MSP作用的关键, 因 此, 在孵育时额外加人适量微气泡可以起到进一步 增强标记的效果. Fan等人 ${ }^{[50]}$ 在气泡表面修饰了具有 靶向性的配体, 能够与细胞表面表达的受体特异性 结合, 气泡在细胞膜上定位后在超声作用下局部穿 孔, 实现单细胞水平上标记量增加的同时能够控制 颗粒在细胞内的分布. 最近, 本研究组 ${ }^{[51]}$ 在微气泡 表面修饰磁性颗粒, 超声作用下观察到超过 $80 \%$ 的 微泡在超声暴露后破裂, 区别于定位在溶酶体, 磁性 颗粒大部分定位在细胞质中, 颗粒引起的毒性显著 降低.

磁场作用是指在细胞与颗粒共孵育时施加磁场 提高标记率. 最初研究发现将SPION与DNA结合, 在 静磁场的作用下观察到SPION摄人增强, 由此DNA 大量进人细胞实现了高效转染, 磁转染技术得到广 泛关注和使用. Smith等人 ${ }^{[52]}$ 在 $350 \mathrm{mT}$ 大小的静磁场
下, 对比了无修饰SPION与穿透肽修饰的SPION标记 细胞的差异, 结果显示标记了穿透肽的SPION在静 磁场作用下标记量显著增多，对内吞的机制分析表 明磁场可能对网格蛋白起到了诱导作用. 对于磁场 增加颗粒吞噬量的机制分析, 有研究通过温度的改 变发现颗粒进人细胞并非被动扩散和磁力牵拉, 而 是一种能量依赖的过程 ${ }^{[53]}$. 此外, 脉冲磁场也被发 现能够促进细胞标记, Lee等人 ${ }^{[54]}$ 合成了一种负载蛋 白质的SPION, 外加高强度的脉冲磁场标记贴壁的 HELA 细胞, 磁场强度为 $0.6 \mathrm{~T}$, 作用 3 次, 每次间隔 $6 \mathrm{~s}$. 结果显示 $24 \mathrm{~h}$ 后脉冲磁场作用下标记量由 1.9 $\mathrm{pg} / \mathrm{cell}$ 提高到 $7.6 \mathrm{pg} / \mathrm{cell}$, 作者将这一现象称为“磁轰 击”, 认为是由于磁性颗粒在脉冲磁场作用下剧烈而 射人细胞质中. 物理场作用时的参数设置尤为重要, 施加的刺激过大会导致细胞死亡, 寻找最合适的标 记条件是研究中的关键任务, 外加场刺激对细胞内 部能量、机械力、膜性质的改变, 以及标记后细胞与 SPION相互作用还有待进一步研究.

\section{2 报告基因转染干细胞}

SPION标记法一个主要的限制是检测到的信号 不能够反映细胞的活性以及生物学特性, 导致检测 的信号存在假阳性. 为了克服直接细胞标记的这些 问题, 研究者开发了一种对细胞进行转染使其表达 某种特定蛋白质用于MRI成像的方法, 所转染的基 因称为报告基因.

目前使用的报告基因主要集中在两类蛋白质， 其中一类蛋白质是通过金属蛋白和金属离子转运蛋 白的过表达以提高细胞内顺磁含量, 从而增强核弛 豫率并在 T1或T2加权MRI中产生高对比度 ${ }^{[55,56]}$, 铁 蛋白报告基因不会被细胞分裂所稀释, 使得它们成 为通过MRI跟踪靶细胞的理想方式. Campan等人 ${ }^{[57]}$ 用人铁蛋白重链作为报告基因在体内跟踪干细胞, 他们用慢病毒载体对猪心脏球体进行转染实现人铁 蛋白重链的过表达, 组织学分析表明铁蛋白过表达 不影响细胞分化. Vande Velde等人 ${ }^{[58]}$ 在啮齿动物脑 中过表达铁蛋白实现 $\mathrm{T} 2 *$ 加权MRI的对比度增强. 有 研究发现通过融合 $\mathrm{L}$ 和 H亚基可以形成一个新的嵌合 铁蛋白分子, 铁负载的增多使细胞表现出显著的 MRI对比增强 ${ }^{[59]}$.

另一类是利用具有大量碱性或酸性氨基酸的蛋 白质，使其和水质子之间产生对比 ${ }^{[60]}$. Gilad等人 ${ }^{[61]}$ 
开发了一种非金属、可降解、富含赖氨酸的蛋白质 (lysine-rich protein, LRP)报告基因, 利用射频辐射非 侵人性地标记与水交换的质子实现细胞活性状况的 区分, 并且在多个细胞分裂后也能够产生恒定的内 源表达水平. 基于化学交换饱和转移 (chemical exchange sturation transfer, CEST)的原理, 富含精氨酸 (47\%精氨酸残基)的人类精蛋白-1也可以由此产生高 磁共振对比度, 由于这种蛋白质形成源于人类基因, 因此具有很好的生物相容性 ${ }^{[62]}$. 最近, Mukherjee等 人 $^{[63]}$ 发现了一种用于MRI成像的新型报告基因: 源 于人的水通道蛋白的基因, 基于增加组织水扩散的 原理, 当含有 $10 \%$ 的细胞表达水通道蛋白就足以增 强造影.

报告基因标记效率关键在于过表达蛋白质量的 多少, 以及这些特殊蛋白质本身性质. 而利用SPION 磁性标记细胞时, 颗粒在摄入后会经历细胞内的降 解代谢, 因此标记效率一定程度上会受到细胞内代 谢的影响, 以下将从 SPION在细胞内的代谢活动方 面进行展述, 进一步对磁性标记的效率进行探讨.

\section{SPION的细胞内代谢}

SPION的细胞内代谢是指在细胞内经历连续的 消化、吸收、外排过程, 是标记后细胞内铁浓度不断 减少的主要原因之一 ${ }^{[64]}$. 因此明确SPION在细胞内 的代谢机制可以在维持细胞活性的同时寻找提高标 记效率的方法. 代谢途径会因内吞途径的不同而有 所区别, 例如, 当内吞依赖网格蛋白介导时, 网格蛋 白内陷形成小泡, 最后大部分经过内体进人溶酶体. 当内吞依赖小窝蛋白介导时, 小窝蛋白由于体积较 小导致颗粒摄取较慢, 形成小泡随后进人到溶酶体、 内质网、内体等多种细胞器中.

\subsection{SPION的胞内降解}

溶酶体是细胞降解SPION的主要细胞器, 可以 对聚合物及无机氧化物进行降解、胞吐, SPION在细 胞内的转运消化是缓慢的过程, 目前研究集中在 SPION在细胞短时程的代谢分析, 而代谢的长时程 监测分析还很缺乏. 通常认为溶酶体将SPION消化 成铁离子, 随后在细胞质内通过Fenton反应催化细胞 内的 $\mathrm{H}_{2} \mathrm{O}_{2}$ 产生· $\mathrm{OH}$, 引发活性氧增加, 由此产生了细 胞毒性进而导致细胞调亡 ${ }^{[65 ~ 67]}$. 另外, SPION的毒性 作用与细胞内微环境相关, Chen等人 ${ }^{[68]}$ 提出 SPION
在不同 $\mathrm{pH}$ 下表现出不同的类酶活性, 如图3所示, 有 别于溶酶体的酸性环境, 细胞质中 SPION由于其中 性环境而表现出过氧化氢酶活性, 催化 $\mathrm{H}_{2} \mathrm{O}_{2}$ 产生 $\mathrm{H}_{2} \mathrm{O}$, 不会产生细胞毒性. 之后, 普鲁士蓝纳米颗粒 (prussian blue nanoparticles, PBNPs)被发现具有更高 类酶活性，不同于 $\mathrm{Fe}_{3} \mathrm{O}_{4}, \mathrm{PBNPs}$ 在不同 $\mathrm{pH}$ 条件下均 具有类超氧化物歧化酶的作用, 可以清除超氧阴离 子自由基, 产生很小的细胞毒性 ${ }^{[69]}$. SPION在细胞内 长时程的代谢跟踪也受到了研究者的关注, 持续监 测的困难主要在于检测方式的缺乏. 最近有研究通 过构建标记有 SPION的干细胞球组织模型对 SPION 在细胞内长时程的代谢进行了定量检测, 对溶酶体 (200 nm) 和模拟溶酶体建立的组织铁代谢模型 $(0.5$ $\mathrm{mm}$ )这两种不同尺度的分析发现：SPION的降解几乎 完全存在于溶酶体, 27 天后溶酶体里几乎不存在 SPION, 降解形成的铁离子分散在细胞质后, 转化为 转铁蛋白, 这种铁元素的回收方式会使铁含量处于 平衡稳定的状态 ${ }^{[70]}$. 在标记细胞时, 除了颗粒对细 胞的影响值得我们关注, 细胞内的复杂环境对 SPION的结构和性质也会产生影响, 例如进人细胞 后由于涂层的降解、生物分子的吸附，颗粒间会产生 聚集 ${ }^{[71]}$, 同时其升温能力、超顺磁性亦会产生不同程 度的减弱 ${ }^{[72]}$.

这些研究对如何减少SPION内吞造成的细胞毒 性具有启发作用. 可能的途径之一是改变颗粒内化 途径, 本研究组 ${ }^{[11]}$ 在微气泡表面修饰磁性颗粒, 利 用MSP对HepG2细胞进行标记, 研究表明超声穿孔

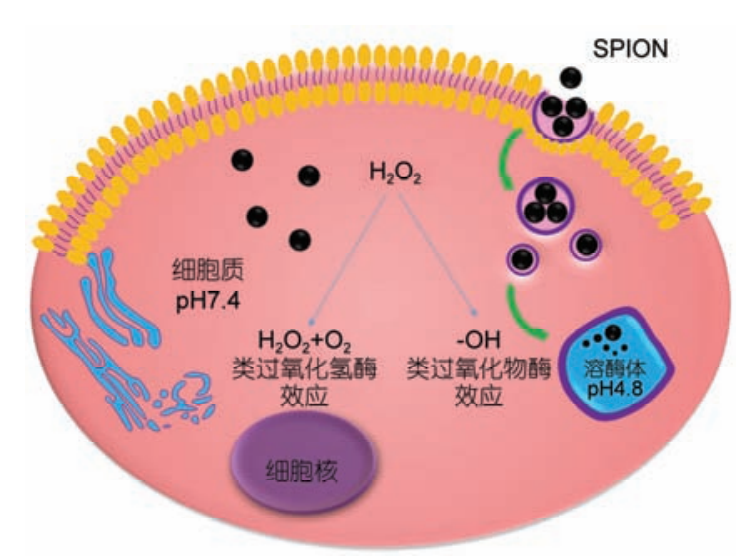

图 3 (网络版彩色)氧化铁颗粒的双类酶效应. Reprinted with permission from Ref. [68], Copyright @ 2012 American Chemical Society Figure 3 (Color online) Dual enzyme-like activities of SPION. Reprinted with permission from Ref. [68], Copyright (C) 2012 American Chemical Society 
后大部分颗粒直接进人细胞质而不是通过内吞摄人, 细胞质内呈中性的酸碱环境使细胞内活性氧水平显 著降低. 另一种途径是通过溶酶体逃逸, 使溶酶体中 的SPION在被分解前释放到细胞质中, 从而减小由 SPION分解引起的氧化应激. 目前研究表明可以通 过两种机制实现溶酶体逃逸, 其一是造成溶酶体内 外的渗透压差, 外部物质内流引起溶酶体涨裂, 例如 聚乙烯亚胺 ${ }^{[73]}$ 、聚乳酸 ${ }^{[74]} 、$ 聚酰胺 ${ }^{[75]}$. 其二是通过 能与膜融合的物质破坏膜结构, 例如流感病毒、黄蜂 肽, 物质氯喹最近也被发现能够用于逃逸溶酶体 ${ }^{[76]}$.

\subsection{SPION外排}

在溶酶体中降解的部分SPION以铁离子的形式排 到细胞质中, 而过剩的SPION将被包裹在囊泡中通过 胞吐的方式排出细胞, SPION外排的过程通常与降解 同步 ${ }^{[77]}$. 研究发现纳米颗粒的尺寸越小越容易被外 排, 14,50 和 $74 \mathrm{~nm}$ 的金纳米颗粒, 胞吐的比例依次为 $35 \%, 10 \%$ 和 $5 \%$. 相同的研究还报道, 细胞对转铁蛋白 包被的棒状纳米颗粒的胞吞作用较弱而胞吐作用较 强, 因此细胞颗粒排泄可以通过大小和形状调制 ${ }^{[78]}$.

除了通过溶酶体的胞吐作用, 细胞在应急状态 下形成的囊泡也能够将细胞内的SPION运送到细胞 外, 紧接着释放出的囊泡会被周围的正常细胞再吞 噬, 形成对其他细胞的再标记 ${ }^{[79]}$. SPION的排出与外 泌体也存在一定联系, 外泌体是多泡体与细胞膜融 合后产生的胞外小囊泡, 直径 40 100 nm. Froeh$\operatorname{lich}^{[64]}$ 提到, 纳米颗粒的增加会引起氧化应激反应, 外泌体会随着细胞内的压力和氧化应激增多而增多, 从而纳米颗粒的增多会导致外泌体数量增加, 由此 假设可以通过这种通路来促进纳米颗粒自身外排. 最近, Busato等人 ${ }^{[80]}$ 利用细胞与SPION共孵育的方式 获取被标记的外泌体, 研究结果表明普通孵育的方 式就能够使排出的外泌体标记上SPION, 也证实了 外泌体确实是一种外排方式.

\section{SPION对干细胞生理功能的影响}

SPION作为干细胞示踪的造影剂, 对于干细胞 来说是一种 “外来物”, 进入细胞内部后参与细胞的 生长代谢, 在这个过程中, 细胞的活性、分化、迁移 等性质的改变影响着细胞移植后在体内的治疗、安全 性等问题 ${ }^{[81 ~ 83]}$, 因此标记后的细胞所具有的性质是 否和正常干细胞一致值得我们关注.
Pongrac等人 ${ }^{[84]}$ 为揭示 SPION对于 NSC 的活性影 响, 发现用SPION标记的NSCs中细胞内谷胱甘肽水 平降低, 超氧化物歧化酶活性降低, 谷胱甘肽过氧化 物酶上调, 线粒体膜电位超极化以及DNA损伤增加. 虽然表面涂层理应能够缓解SPION对于细胞的毒性 作用, 但结果显示实验中所有SPION类型(未涂覆、涂 覆有 D-甘露糖或涂覆有聚L-赖氨酸)都会影响 NSC, 这表明它们的主要细胞靶标是线粒体稳态, 同时, 最 近有研究发现在一些间充质干细胞中检测到类似于 调亡小体的结构 ${ }^{[85]}$. 针对迁移和分化方面的影响, Magnitsky等人 ${ }^{[86]}$ 利用NSC和C17.2进行了研究, 结果 显示用SPION标记后的原代NSC迁移能力受到一定 程度限制, 而对 C17.2细胞迁移能力影响不大. 约翰 霍普金斯大学Walczak研究组 ${ }^{[87]}$ 也发现标记后神经 干细胞运动性的降低. 干细胞的分化影响目前主要 集中在成骨分化的变化, 之前的一些研究表明细胞 在磁性标记后会抑制软骨形成 ${ }^{[88,89]}$. 本研究组 ${ }^{[90]}$ 发 现SPION可以激活经典的有丝分裂原活化蛋白激酶 信号通路，从而调节下游信号促进人类骨源性间充 质干细胞在体外的成骨分化.

目前, 对于干细胞行为和性质的影响认知还不 完全, 原因之一在于对其干性改变的分析没有系统 的方法和手段, 另一方面, 有关细胞示踪方面的研 究, 研究者通常将重点放在示踪效果, 而往往对细胞 性质的影响有所忽视. 然而, 对干细胞性质的研究能 够起到反馈的作用, 通过检测细胞的变化可以反向 优化示踪剂以及标记手段.

\section{4 总结与展望}

干细胞治疗是当前最有潜力的组织修复方式, 现有的研究状况表明目前主要还停留在实验阶段, 临床研究比较缺乏, 大部分临床研究主要针对脊髓 损伤和脑损伤两类模型, 标记的干细胞移植后成像 信号显示大约可以维持3周, 7周之后成像信号基本消 失 ${ }^{[91]}$, 持续数周的MRI监测结果同时表明移植的干 细胞能够自主向损伤部位迁移 ${ }^{[92]}$. Janowski等人 ${ }^{[93]}$ 针 对紧幼儿脑损伤模型将SPION标记的神经细胞经过 脑室注射如脑内, 通过外部施加静磁场, 观察到细胞 顺着磁场方向发生定向迁移.

通过一系列体内外实验验证, MRI成像是现在公 认的比较合适的一种体外示踪方式, 因此本文对现 有的干细胞磁性标记方式, 以及细胞内代谢、增殖分 
化影响进行了描述, 并且对不同标记类型的优缺点 进行了分析. 应用于临床的标记物一定要保证具有 很小的毒性、良好的生物相容性, 在体内维持长循环 以及高质量的成像效果. 研究证明基于颗粒表面修 饰方式具有高标记量的特点, 而物理场作用特点在 于快速标记, 结合两者的优势将是以后标记方式的 发展趋势.

结合本研究组即将开展的工作, 对干细胞的磁 性标记提出了以下思考: 对于提高细胞内标记量, 一 方面要增强细胞对颗粒的吞噬, 另一方面要减少细 胞对颗粒的降解、外排. 标记的增加有赖于新型转染
剂、报告基因的开发以及与物理场结合的协同作用, 通过内吞途经摄人的SPION大部分会进人溶酶体分 解, 因此加人能使颗粒从溶酶体中逃逸的材料或许 可以一定程度上减少颗粒的分解, 实现细胞内更长 时间的滞留. 同时, 细胞外标记的方式也引起了关 注, 现有的细胞外标记磁珠方式多用于细胞分选 ${ }^{[94]}$, 细胞外标记优势在于不涉及胞内的降解，但是用于体 内的问题在于细胞上负载了磁性颗粒在体内很容易被 内皮系统识别, 引起巨噬细胞吞噬, 但我们猜想如果 在磁性颗粒外部修饰细胞自身来源的物质是否可以避 免巨噬细胞的清除, 这有待更进一步的实验考证.

\section{参考文献}

1 Czarzasta J, Habich A, Siwek T, et al. Stem cells for ALS: An overview of possible therapeutic approaches. Int J Develop Neuroscit, 2017, 57: 46-55

2 Becker A J, Till J E, Mcculloch E A. Cytological demonstration of clonal nature of spleen colonies derived from transplanted mouse marrow cells. Nature, 1963, 197: 452

3 Lin C L, Ho Y. A bibliometric analysis of publications on pluripotent stem cell research. Cell J, 2015, 17: 59-70

4 Zeng W, Xiao J, Zheng G, et al. Antioxidant treatment enhances human mesenchymal stem cell anti-stress ability and therapeutic efficacy in an acute liver failure model. Sci Rep, 2015, 5: 11100

5 Petrou P, Gothelf Y, Argov Z, et al. Safety and clinical effects of mesenchymal stem cells secreting neurotrophic factor transplantation in patients with amyotrophic lateral sclerosis results of phase 1/2 and 2a clinical trials. JAMA Neurol, 2016, 73: 337-344

6 Qi Y, Feng G, Huang Z, et al. The application of super paramagnetic iron oxide-labeled mesenchymal stem cells in cell-based therapy. Mol Biol Rep, 2013, 40: 2733-2740

7 Singh J P. Enabling technologies for homing and engraftment of cells for therapeutic applications. JACC Cardiovasc Intev, 2009, 2: 803-804

8 Li L, Jiang W, Luo K, et al. Superparamagnetic iron oxide nanoparticles as mri contrast agents for non-invasive stem cell labeling and tracking. Theranostics, 2013, 3: 595-615

9 Phanapavudhikul P, Shen S, Ng W K, et al. Formulation of $\mathrm{Fe}_{3} \mathrm{O}_{4} /$ acrylate co-polymer nanocomposites as potential drug carriers. Drug Deliv, 2008, 15: 177-183

10 Wilhelm C, Bal L, Smirnov P, et al. Magnetic control of vascular network formation with magnetically labeled endothelial progenitor cells. Biomaterials, 2007, 28: 3797-3806

11 Hauser A K, Mitov M I, Daley E F, et al. Targeted iron oxide nanoparticles for the enhancement of radiation therapy. Biomaterials, 2016, 105: 127-135

12 Peng X, Qian X, Mao H, et al. Targeted magnetic iron oxide nanoparticles for tumor imaging and therapy. Int J Nanomed, 2008, 3: 311-321

13 Clay N, Baek K, Shkumatov A, et al. Flow-mediated stem cell labeling with superparamagnetic iron oxide nanoparticle clusters. ACS Appl Mater Interfaces, 2013, 5: 10266-10273

14 Sahay G, Alakhova D Y, Kabanov A V. Endocytosis of nanomedicines. J Control Release, 2010, 145: 182-195

15 Shi Z, Neoh K G, Kang E T, et al. (Carboxymethyl)chitosan-modified superparamagnetic iron oxide nanoparticles for magnetic resonance imaging of stem cells. ACS Appl Mater Interfaces, 2009, 1: 328-335

16 Laurent S, Forge D, Port M, et al. Magnetic iron oxide nanoparticles: Synthesis, stabilization, vectorization, physicochemical characterizations, and biological applications, Chem Rev, 2010, 110: 2574

17 Paweletz N. Walther flemming: Pioneer of mitosis research. Nat Rev Mol Cell Bio, 2001, 2: 72

18 Zhang K, Fang H, Chen Z, et al. Shape effects of nanoparticles conjugated with cell-penetrating peptides (Hiv Tat Ptd) on CHO cell uptake. Bioconjugate Chem, 2008, 19: 1880-1887

19 Qiao R, Qiao H, Zhang Y, et al. Molecular imaging of vulnerable atherosclerotic plaques in vivo with osteopontin-specific upconversion nanoprobes. ACS Nano, 2017, 11: 1816-1825 
20 Kanwar R K, Chaudhary R, Tsuzuki T, et al. Emerging engineered magnetic nanoparticulate probes for molecular MRI of atherosclerosis: How far have we come? Nanomedicine, 2012, 7: 899-916

21 Zhao P, Cao M, Song L, et al. Downregulation of MIM protein inhibits the cellular endocytosis process of magnetic nanoparticles in macrophages. RSC Adv, 2016, 6: 96635-96643

22 Lee D H, Kang M, Lee H J, et al. Enhanced cellular uptake of silica-coated magnetite nanoparticles compared with PEG-coated ones in stem cells. J Nanosci Nanotechnol, 2015, 15: 5512-5519

23 Barrow M, Taylor A, Murray P, et al. Design considerations for the synthesis of polymer coated iron oxide nanoparticles for stem cell labelling and tracking using MRI. Chem Soc Rev, 2015, 44: 6733-6748

24 Reddy A M, Kwak B K, Shim H J, et al. In vivo tracking of mesenchymal stem cells labeled with a novel chitosan-coated superparamagnetic iron oxide nanoparticles using 3.0 T MRI. J Korean Med Sci, 2010, 25: 211-219

25 Kaklotar D, Agrawal P, Abdulla A, et al. Transition from passive to active targeting of oral insulin nanomedicines: Enhancement in bioavailability and glycemic control in diabetes. Nanomedicine, 2016, 11: 1465-1486

26 Matsumoto Y, Chen R, Anikeeva P, et al. Engineering intracellular biomineralization and biosensing by a magnetic protein. Nat Commun, 2015, 6: 8721

27 Wang Y J, Xuan S, Port M, et al. Recent advances in superparamagnetic iron oxide nanoparticles for cellular imaging and targeted therapy research. Curr Pharm Design, 2013, 19: 6575-6593

28 Bakhru S H, Altiok E, Highley C, et al. Enhanced cellular uptake and long-term retention of chitosan-modified iron-oxide nanoparticles for MRI-based cell tracking. Int J Nanomedicine, 2012, 7: 4613-4623

29 Lewin M, Carlesso N, Tung C H, et al. Tat peptide-derivatized magnetic nanoparticles allow in vivo tracking and recovery of progenitor cells. Nat Biotechnol, 2000, 18: 410-414

30 Josephson L, Tung C H, Moore A, et al. High-efficiency intracellular magnetic labeling with novel superparamagnetic-tat peptide conjugates. Bioconjugate Chem, 1999, 10: 186-191

31 Jackson J, Chapon C, Jones W, et al. In vivo multimodal imaging of stem cell transplantation in a rodent model of parkinson's disease. J Neurosci Meth, 2009, 183: 141-148

32 Jeong H, Lee B C, Ahn B, et al. Development of drugs and technology for radiation theragnosis. Nucl Eng Technol, 2016, 48: 597-607

33 Yue Z, Wei W, Lv P, et al. Surface charge affects cellular uptake and intracellular trafficking of chitosan-based nanoparticles. Biomacromolecules, 2011, 12: 2440-2446

34 Bulte J, Douglas T, Witwer B, et al. Magnetodendrimers allow endosomal magnetic labeling and in vivo tracking of stem cells. Nat Biotechnol, 2001, 19: 1141-1147

35 Frank J A, Miller B R, Arbab A S, et al. Clinically applicable labeling of mammalian and stem cells by combining superparamagnetic iron oxides and transfection agents. Radiology, 2003, 229: 610

36 Maurer J, Haselbach S, Klein O, et al. Analysis of the complex formation of heparin with protamine by light scattering and analytical ultracentrifugation: Implications for blood coagulation management. J Am Chem Soc, 2011, 133: 1134-1140

37 Bryant L H J, Kim S J, Hobson M, et al. Physicochemical characterization of ferumoxytol, heparin and protamine nanocomplexes for improved magnetic labeling of stem cells. Nanomed Nanotechnol Biol Med, 2017, 13: 503-513

38 Kim H, Dae H, Park C, et al. A highly sensitive magnetite nanoparticle as a simple and rapid stem cell labelling agent for mri tracking. J Mater Chem, 2011, 21: 7742-7747

39 Andreas K, Georgieva R, Ladwig M, et al. Highly efficient magnetic stem cell labeling with citrate-coated superparamagnetic iron oxide nanoparticles for MRI tracking. Biomaterials, 2012, 33: 4515-4525

40 Wang Y J, Quercy-Jouvet T, Wang H, et al. Efficacy and durability in direct labeling of mesenchymal stem cells using ultrasmall superparamagnetic iron oxide nanoparticles with organosilica, dextran, and PEG coatings. Materials, 2011, 4: 703-715

41 Coradin T, Lopez P J. Biogenic silica patterning: Simple chemistry or subtle biology? ChemBioChem, 2003, 4: 251-259

42 Strand B L, Ryan L, Veld P I, et al. Poly-L-lysine induces fibrosis on alginate microcapsules via the induction of cytokines. Cell Transplant, 2001, 10: 263-275

43 Walczak P, Kedziorek D A, Gilad A A, et al. Instant MR labeling of stem cells using magnetoelectroporation. Magn Reson Med, 2005, 54: 769-774

44 Walczak P, Ruiz-Cabello J, Kedziorek D A, et al. Magnetoelectroporation: Improved labeling of neural stem cells and leukocytes for cellular magnetic resonance imaging using a single FDA-approved agent. Nanomed Nanotechnol, 2006, 2: 89-94

45 Kim T, Momin E, Choi J, et al. Mesoporous silica-coated hollow manganese oxide nanoparticles as positive T1 contrast agents for labeling and mri tracking of adipose-derived mesenchyrnal stem cells. J Am Chem Soc, 2011, 133: 2955-2961

46 Bao S P, Thrall B D, Miller D L. Transfection of a reporter plasmid into cultured cells by sonoporation in vitro. Ultrasound Med Biol, 
1997, 23: 953-959

Qiu B, Xie D, Walczak P, et al. Magnetosonoporation: Instant magnetic labeling of stem cells. Magn Reson Med, 2010, 63: 1437-1441

Xie D, Qiu B, Walczak P, et al. Optimization of magnetosonoporation for stem cell labeling. NMR Biomed, 2010, 23: 480-484

Lei H, Nan X, Wang Z, et al. Stem cell labeling with superparamagnetic iron oxide nanoparticles using focused ultrasound and magnetic resonance imaging tracking. J Nanosci Nanotechnol, 2015, 15: 2605-2612

Fan Z, Liu H, Mayer M, et al. Spatiotemporally controlled single cell sonoporation. Proc Natl Acad Sci USA, 2012, 109: 16486-16491

Yang F, Li M, Cui H, et al. Altering the response of intracellular reactive oxygen to magnetic nanoparticles using ultrasound and microbubbles. Sci China Mater, 2015, 58: 467-480

Smith C M, de la Fuente J, Pelaz B, et al. The effect of static magnetic fields and tat peptides on cellular and nuclear uptake of magnetic nanoparticles. Biomaterials, 2010, 31: 4392-4400

Liu Q, Zhang J, Xia W, et al. Towards magnetic-enhanced cellular uptake, MRI and chemotherapeutics delivery by magnetic mesoporous silica nanoparticles. J Nanosci Nanotechnol, 2012, 12: 7709-7715

Lee C, Chen C, Chung T, et al. Cellular uptake of protein-bound magnetic nanoparticles in pulsed magnetic field. J Nanosci Nanotechnol, 2010, 10: 7965-7970

Patrick P S, Rodrigues T B, Kettunen M I, et al. Development of Timd2 as a reporter gene for MRI. Magn Reson Med, 2016, 75: 1697-1707

Kim H S, Woo J, Choi Y, et al. Noninvasive MRI and multilineage differentiation capability of ferritin-transduced human mesenchymal stem cells. NMR Biomed, 2015, 28: 168-179

Campan M, Lionetti V, Aquaro G D, et al. Ferritin as a reporter gene for in vivo tracking of stem cells by $1.5 \mathrm{~T}$ cardiac mri in a rat model of myocardial infarction. Am J Physiol Heart C, 2011, 300: H2238-H2250

Vande Velde G, Rangarajan J R, Toelen J, et al. Evaluation of the specificity and sensitivity of ferritin as an MRI reporter gene in the mouse brain using lentiviral and adeno-associated viral vectors. Gene Ther, 2011, 18: 594-605

Iordanova B, Ahrens E T. In vivo magnetic resonance imaging of ferritin-based reporter visualizes native neuroblast migration. Neuroimage, 2012, 59: 1004-1012

Airan R D, Bar-Shir A, Liu G, et al. MRI biosensor for protein kinase a encoded by a single synthetic gene. Magn Reson Med, 2012, 68: 1919-1923

Gilad A A, Mcmahon M T, Walczak P, et al. Artificial reporter gene providing MRI contrast based on proton exchange. Nat Biotechnol, 2007, 25: 217-219

Bar-Shir A, Liu G, Chan K W Y, et al. Human protamine-1 as a MRI reporter gene based on chemical exchange. ACS Chem Biol, 2014, 9: $134-138$

Mukherjee A, Wu D, Davis H C, et al. Non-invasive imaging using reporter genes altering cellular water permeability. Nat Commun, 2016, 7: 13891

Froehlich E. Cellular elimination of nanoparticles. Environ Toxicol Phar, 2016, 46: 90-94

Feng X, Mao G Y, Bu F X, et al. Controlled synthesis of monodisperse $\mathrm{CoFe}_{2} \mathrm{O}_{4}$ nanoparticles by the phase transfer method and their catalytic activity on methylene blue discoloration with $\mathrm{H}_{2} \mathrm{O}_{2}$. J Magn Magn Mater, 2013, 343: 126-132

Naqvi S, Samim M, Abdin M Z, et al. Concentration-dependent toxicity of iron oxide nanoparticles mediated by increased oxidative stress. Int J Nanomed, 2010, 5: 983-989

Arbab A S, Wilson L B, Ashari P, et al. A model of lysosomal metabolism of dextran coated superparamagnetic iron oxide (spio) nanoparticles: Implications for cellular magnetic resonance imaging. NMR Biomed, 2005, 18: 383-389

Chen Z, Yin J J, Zhou Y T, et al. Dual enzyme-like activities of iron oxide nanoparticles and their implication for diminishing cytotoxicity. ACS Nano, 2012, 6: 4001-4012

Zhang W, Hu S, Yin J, et al. Prussian blue nanoparticles as multienzyme mimetics and reactive oxygen species scavengers. J Am Chem Soc, 2016, 138: 5860-5865

Mazuel F, Espinosa A, Luciani N, et al. Massive intracellular biodegradation of iron oxide nanoparticles evidenced magnetically at singleendosome and tissue levels. ACS Nano, 2016, 10: 7627-7638

Levy M, Wilhelm C, Luciani N, et al. Nanomagnetism reveals the intracellular clustering of iron oxide nanoparticles in the organism. Nanoscale, 2011, 3: 4402-4410

Kolosnjaj-Tabi J, Lartigue L, Javed Y, et al. Biotransformations of magnetic nanoparticles in the body. Nano Today, 2016, 11: 280-284

Yamada H, Loretz B, Lehr C. Design of starch-graft-PEI polymers: An effective and biodegradable gene delivery platform. Biomacromolecules, 2014, 15: 1753-1761

Liu G, Ma S, Li S, et al. The highly efficient delivery of exogenous proteins into cells mediated by biodegradable chimaeric polymersomes. Biomaterials, 2010, 31: 7575-7585 
75 Higuchi Y, Wu C, Chang K, et al. Polyamidoamine dendrimer-conjugated quantum dots for efficient labeling of primary cultured mesenchymal stem cells. Biomaterials, 2011, 32: 6676-6682

76 Harhaji-Trajkovic L, Arsikin K, Kravic-Stevovic T, et al. Chloroquine-mediated lysosomal dysfunction enhances the anticancer effect of nutrient deprivation. Pharm Res Dordr, 2012, 29: 2249-2263

77 Chu Z, Huang Y, Tao Q, et al. Cellular uptake, evolution, and excretion of silica nanoparticles in human cells. Nanoscale, 2011, 3: 3291-3299

78 Chithrani D B. Intracellular uptake, transport, and processing of gold nanostructures. Mol Membr Biol, 2010, 27: 299-311

79 Luciani N, Wilhelm C, Gazeau F. The role of cell-released microvesicles in the intercellular transfer of magnetic nanoparticles in the monocyte/macrophage system. Biomaterials, 2010, 31: 7061-7069

80 Busato A, Bonafede R, Bontempi P, et al. Magnetic resonance imaging of ultrasmall superparamagnetic iron oxide-labeled exosomes from stem cells: A new method to obtain labeled exosomes. Int J Nanomed, 2016, 11: 2481-2490

81 Kallur T, Farr T D, Boehm-Sturm P, et al. Spatio-temporal dynamics, differentiation and viability of human neural stem cells after implantation into neonatal rat brain. Eur J Neurosci, 2011, 34: 382-393

82 Ramos-Gomez M, Martinez-Serrano A. Tracking of iron-labeled human neural stem cells by magnetic resonance imaging in cell replacement therapy for parkinson's disease. Neural Regen Res, 2016, 11: 49-52

83 Arbab A S, Yocum G T, Rad A M, et al. Labeling of cells with ferumoxides-protamine sulfate complexes does not inhibit function or differentiation capacity of hematopoietic or mesenchymal stem cells. NMR Biomed, 2005, 18: 553-559

84 Pongrac I M, Pavicic I, Milic M, et al. Oxidative stress response in neural stem cells exposed to different superparamagnetic iron oxide nanoparticles. Int J Nanomed, 2016, 11: 1701

85 Silva L H A, Da Silva J R, Ferreira G A, et al. Labeling mesenchymal cells with DMSA-coated gold and iron oxide nanoparticles: Assessment of biocompatibility and potential applications. J Nanobiotechnol, 2016, 14: 59

86 Magnitsky S, Walton R M, Wolfe J H, et al. Magnetic resonance imaging detects differences in migration between primary and immortalized neural stem cells. Acad Radiol, 2008, 15: 1269-1281

87 Berman S M C, Kshitiz, Wang C J, et al. Cell motility of neural stem cells is reduced after spio-labeling, which is mitigated after exocytosis. Magn Reson Med, 2013, 69: 255-262

88 Bulte J, Kraitchman D L, Mackay A M, et al. Chondrogenic differentiation of mesenchymal stem cells is inhibited after magnetic labeling with ferumoxides. Blood, 2004, 104: 3410-3412

89 Kostura L, Kraitchman D L, Mackay A M, et al. Feridex labeling of mesenchymal stem cells inhibits chondrogenesis but not adipogenesis or osteogenesis. Nmr Biomed, 2004, 17: 513-517

90 Wang Q, Chen B, Cao M, et al. Response of MAPK pathway to iron oxide nanoparticles in vitro treatment promotes osteogenic differentiation of hbmscs. Biomaterials, 2016, 86: 11-20

91 Zhang W Y, Ebert A D, Narula J, et al. Imaging cardiac stem cell therapy: Translations to human clinical studies. J Cardiovasc Transl, 2011, 4: 514-522

92 Karussis D, Karageorgiou C, Vaknin-Dembinsky A, et al. Safety and immunological effects of mesenchymal stem cell transplantation in patients with multiple sclerosis and amyotrophic lateral sclerosis. Archives Neurol, 2010, 67: 1187-1194

93 Janowski M, Walczak P, Kropiwnicki T, et al. Long-term MRI cell tracking after intraventricular delivery in a patient with global cerebral ischemia and prospects for magnetic navigation of stem cells within the CSF. PLoS One, 2014, 9: e97631

94 Handgretinger R. Isolation and transplantation of highly purified autologous peripheral blood CD34+ progenitors in neuroblastoma. Bone Marrow Transpl, 1998, 223: S67 
Summary for “干细胞的磁性标记研究进展”

\title{
Recent progress in magnetic labeling for stem cell
}

\author{
YE DeWen, WANG QiWei, ZHANG WeiGuo, SUN JianFei \& GU Ning* \\ School of Biological Science and Medical Engineering, Southeast University, Nanjing 210096, China \\ *Corresponding author, E-mail: guning@seu.edu.cn
}

Stem cells are cells with self-renewal and multiple differentiation direction characters. Variety of studies have shown that stem cells have broad prospects in tissue repair and regenerative medicine. However, some aspects of stem cells in vivo transplantation developed slowly, for instance, distribution, activity, differentiation direction and mechanism, which have restricted the development of stem cell therapy. Thus, exploring novel technologies for tracing stem cell in vivo will play a vital role in solving the above scientific problems. When stem cell are labeled magnetically, we can obtain a noninvasive, safe, sustained and dynamic tracing in vivo by combining with magnetic resonance imaging (MRI). In addition, major factors influencing the efficiency of in vivo trace contain cellular content of magnetic material, the different methods for magnetic labeling and the maintenance of cell activity.

Here, we review the recent progresses on different ways for magnetic labeling stem cell, the metabolism of magnetic substances and the impact on cell systematically. For magnetic labeling, one of the major aspects focuses on labelling stem cells with magnetic materials like superparamagnetic iron oxide nanoparticles (SPION). Surface modification of SPION and exerting additional physical field when stem cell incubated with SPION both have enhanced label efficiency, however, it could not reflect the actual cellular activity in vivo which might bring out false-positive results by using this approach. Therefore, the use of reporter gene is developed, which could provide strong tissue contrast after stable transfection. However, whether the transfection alters stem cell properties remains to be proved. In addition, after SPION is uptaken by cells, the labeling efficiency will be decreased by a series intracellular degradation and metabolism, most particles is degraded by lysosomes into iron ions which could not provide effective MRI imaging. On the other hand, the cellular reactive oxygen species (ROS) level will increase substantially through Fenton reaction which has toxic effect on cell activity. Thus, SPION degradation in lysosome shows a huge negative influence on tracing, which could not be ignored. Recent research reveals that cell properties such as migration and differentiation will be altered after labeling with SPION.

By analyzing the working principles of existing label technology, we cover a detailed outlook on improving magnetic stem cell labeling. We prospect that two momentous issues should be considered to achieve efficient cell labeling and long-term tracing: Phagocytosis and metabolic pathway. More advanced methods ought to be developed to realize secure, rapid, massive cell label. Meanwhile, regulation of intracellular metabolism pathway is also critical for long-term cell tracing. To some extent, coating materials of SPION that allows particles to escape from lysosomes degradation will allow SPION longtime retention in cell. Moreover, labeling SPION extracellular also attracts our attention and its advantage is that SPION labelled on external of plasma membrane could not only be imaged, but also be escaped from cellular metabolism.

stem cell tracing, stem cell labeling, magnetic resonance imaging, magnetic iron oxide nanoparticle, intracellular iron metabolism

doi: 10.1360/N972017-00458 